\title{
Architecture Design of High Safety Helicopter Flight Control System with Direct Control Mode
}

\author{
Liqiang Teng, Wenshan Wang, and Yining Liu
}

\begin{abstract}
The complex Electronic Control Unit (ECU) is the core of helicopter Flight Control System (FCS), which can cause flight accident when the FCS redundancy is degraded quickly due to its failure, or when ECU fails simultaneously due to common mode failure. Accordingly, a $3 \times 2$ redundant helicopter FCS architecture with direct control mode is designed, by comparing and analyzing the FCS architectural characteristics of typical international advanced NH-90 and S-92 helicopters. In primary control mode, this FCS can fail-operate twice, and ensure level 1 handling qualities. After three failures, the FCS switches to direct control mode and can still tolerate once failure, and the handling qualities changed to level 2. Meanwhile, non-similar design is used to avoid ECU's common mode failure. Finally, the safety of primary control mode is analyzed by fault tree analysis. This paper can provide reference for the FCS architecture design of medium and large helicopters.
\end{abstract}

L. Teng $(\bowtie) \cdot$ W. Wang $\cdot$ Y. Liu

AVIC Qing'an Group Co.Ltd., Xi'an 710077, China

e-mail: liqiangteng@126.com 